\title{
Conditional-outcome choice behavior in squirrel monkeys
}

KENNETH F. GREEN AND JOHN W. MOORE

UNIVERSITY OF MASSACHUSETTS, AMHERST

Six naive squirrel monkeys were given conditional-outcome choice training with two equally-often rewarded alternatives. One was a soluble two-choice color discrimination while the other was a pair of identically-colored stimuli. At regular intervals the monkeys were allowed to choose between them. Two Ss were trained on only one pair of problems, and one of these $S$ s displayed a temporary preference for the conditionaloutcome alternative.

Will an S prefer an alternative where it can control reward, an alternative where reward is under E's control, or will there be no preference? Logan (1962) devised the conditional-outcome choice situation as a means of attacking this question. In a conditionaloutcome choice situation an $S$ is trained concurrently on two equally-often rewarded alternatives. One alternative (conditional) contains a contingency for obtaining reward and the other (nonconditional) does not. Every so often $\mathrm{S}$ is allowed to choose between alternatives. Logan applied this situation in seven studies using rats in double alleys and block-eight mazes and in four cases found preferences for one or the other of the alternatives. The preferences were not strong, and Logan attributed them to chance, to delay of reward, or to an artifact of his reward-matching procedure. The artifact was a lag effected by delivering nonconditional reward in one block of trials with the frequency that $S$ had earned conditional reward in the previous block of trials. Logan concluded that "there is no preference between a conditional-outcome alternative and one with the same frequency of reward uncorrelated with performance" (Logan, 1962, pp. 475-476).

The present experiment attempted to determine whether this conclusion applied to primates in a situation designed to take advantage of the superiority of these animals to rats in ability to solve problems (Warren, 1965) and in responsiveness to visual stimuli. Thus, squirrel monkeys were tested in a situation where the conditional alternative was a two-choice discrimination (two differentiable stimuli) and the nonconditional alternative was an insoluble two-choice problem (two identical stimuli). Neither the conditional nor the nonconditional alternative was associated with a given side of the apparatus, and a new technique for matching nonconditional rewards to conditional performance was introduced. In addition, because one of Logan's studies suggested that a preference was likely to occur during the acquisition phase of a discrimination problem, some monkeys were allowed to solve only to a lax criterion before new problems were introduced.

\section{Method}

The apparatus has been described elsewhere (Green, Moore, \& Sargent, 1966). Briefly, it consisted of a sheet-metal cube with a pair of display panels at one end. Opaque and transparent guillotine screens were used to cover the stimulus holes in the upper half of the display panels, but they did not cover the hole giving access to a delivery tray centered lower in each panel. The panels were white, the opayue screens were gray, and the remainder of the apparatus was black

Eight naive adult squirrel monkeys were trained to press the stimulus panels and to receive reward from the delivery cups. Prior to experimental training all Ss were given a test for color preference (Green et al, 1966).

Experimental training was given each day according to any of a set of 1042 -trial sequences. The sequences specified the order presentation of the six daily choice trials and the three or nine trials between choices on which the Ss were forced to each alternative. The sequences were arranged such that each $\mathbf{S}$ each day responded an equal number of times to each problem and each side of the apparatus. Also denoted was the number of conditional trials ( 4 or 10 ) to be examined for purposes of matching nonconditional rewards to conditional performance. The noncorrection technique was used for all problems.

Nonconditional reward was matched to conditional performance by two methods. Logan's "frequency" procedure was used for one group of animals. A probabilistic procedure was used for a second group of animals wherein the most recent conditional performance was examined and a random device employed (a marble was drawn from an urn or a Lehigh Valley Model 1485 random generator was activated) to determine whether reward would be delivered on any given nonconditional trial.

Two males and two females were tested according to a successive-problem procecture wherein a new set of problems was introduced as soon as a criterion of 14 correct responses within 15 consecutive conditional trials was reached. The males were tested for 70 days and the females for 35 days; three Ss received reward matching by the probabilistic procedure and one by the frequency procedure. Two other males (Anton and Gustav) were trained for 50 days on a single set of problems and for an additional 20 days according to the successive-problem procedure; one of these Ss received reward matching by the probabilistic procedure and the other by the frequency procedure. Two additional females received 42 con- 
ditional-problem trials each day for 20 days under the successive-problem procedure. These Ss served as controls for the possible interference with the development of observing responses (Stollnitz, 1965) effected by presentation of the nonconditional with the conditional problem.

\section{Results}

A problem was designated as genuinely preferred if it was chosen (a) on a least four of the six daily choice trials and (b) at least once when it appeared on the side of the apparatus opposite that preferred by $S$. Application of these criteria showed that genuine preferences occurred on 44 (15.4\%) of the 286 experimental days and within these days on $229(10.8 \%)$ of the 2117 choice trials.

The single-problem monkeys solved the conditional problem rapidly, and for the remainder of the $\mathbf{5 0}$ days on this problem combination the Ss followed the correct stimulus on the conditional alternative and position responded on the nonconditional. Anton displayed a preference on 53 choice trials, thus accounting for $23 \%$ of the genuine preferences of all Ss. The preference was for the conditional alternative, and it was displayed on seven of the first eight days of highlevel conditional performance and on three later days. Under the successive-problem procedure, to which these Ss were switched on the 51st day, neither animal solved the new conditional problem. On one day, however, Gustav displayed a preference for the conditional alternative. Gustav's conditional performance on this day exceeded $60 \%$ correct.

The successive-problem monkeys displayed genuine preferences on $172(75 \%)$ choice trials, but less than $40 \%$ of these preferences occurred at times when conditional performance exceeded $60 \%$ correct and fewer still occurred when the Ss were responding differentially on the two alternatives. The preferences of these monkeys appeared to be related to color in that the selected alternatives were found on $162(94 \%)$ of these trials to contain stimuli which had been shown by a color preference test (Green et al, 1966) to be more preferred than the stimuli in the other alternatives.

Neither of the two control Ss solved problems more efficiently than the better experimental Ss. It thus appears that presentation of the nonconditional problem did not necessarily retard formation of observing responses appropriate to correct conditional responding.

No differential effect on choice behavior was noted for the frequency and probabilistic procedures for matching reward.

\section{Discussion}

Logan (1960) suggested that animals in any situation should response in a manner consistent with maximum reward and minimum effort. Perhaps the most efficient way to respond in the present situation would have been to follow the correct stimulus on the conditional alternative and to position respond on the nonconditional. Given this (or some other) type of differential responding, it would be evident that the monkeys discriminated between alternatives. If it is argued that an $S$ must discriminate between alternatives before meaningful choices between them can be made, then it becomes apparent that the results of only the two single-problem monkeys are relevant to the problem at hand. One of these Ss temporarily preferred the conditional alternative, where reward was under his control. In contrast, when the successive-problem Ss preferred an alternative they selected on the basis of preferred colors rather than reward control. It is apparent that the lax solution criterion minimized the number of choices allowed at times when these Ss were discriminating between alternatives. That is, an $\mathrm{S}$ no sooner attained high-level conditional performance and began responding differentially on the two alternatives than a new set of problems was introduced and the $S$ had to learn anew which alternative contained the contingency.

It should be pointed out that rats in Logan's (1962) situations chose a problem prior to encountering the stimuli within the problems, whereas on choice trials in the present experiment the four stimuli of the two alternatives were presented simultaneously. Nevertheless, in light of the preference for the rewardcontrol alternative shown by one of the single-problem monkeys, and the finding by Weir (1965) that children prefer predictable to nonpredictable reward, it is suggested that Logan's conclusion may not apply to primates.

\section{References}

Green, K. F., Moore, J. W., \& Sargent, T. D. Color preference in squirrel monkeys (Saimiti sciureus). Psychon. Sci., 1966 , 367-368.

Logan, F. A. Incentive. New Haven: Yale. 1960.

Logan, F. A. Conditional-outcome choice behavior in rats. Psychol. Rev., 1962, 69, 467476.

Stollnitz, F. Spatial variables, observing responses, and discrimination learning sets. Psychol. Rev., 1965, 72, 247-261.

Warren, J. M. Primate learning in comparative perspective. In A. M. Schrier, H. F. Harlow, and F. Stollnitz (Eds.), Behavior of nonhuman primates. Vol. I. New York: Academic Press. 1965.

Weir, M. W. Children's behavior in a two-choice task as a function of patterned reinforcement following forced-choice trials. $J$. exp. child Psychol., 1965, 2, 85-91. 